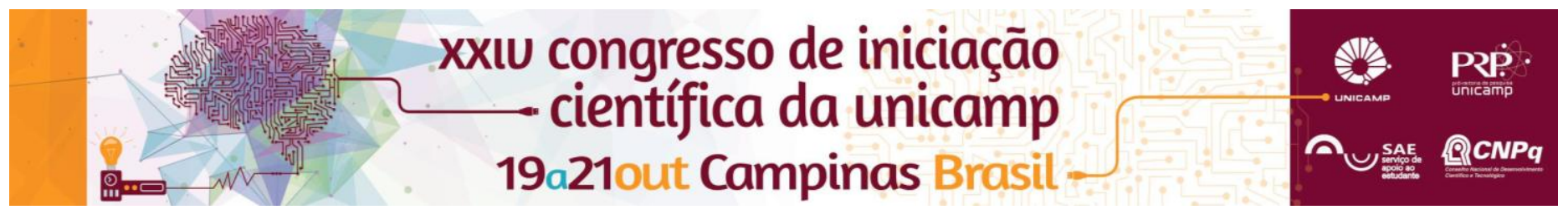

\title{
Fatores socio-demográficos e Saúde Geral em Homens: SM e DM2
}

\author{
Autores: Castro, E.R*; Fortunato,PLM .Orientação: de O. Regina,MC
}

\section{Resumo}

Há poucos estudos sobre o estado de saúde de homens com síndrome metabólica e diabetes tipo 2 . Amostra: 48 homens. Se renda, escolaridade, estado civil, exercícios físicos e dieta associam-se à quadro clinico.Hbgli <6.5:prevalência de não casados, renda menor, escolaridade maior, maior frequência de exercícios, sem dieta; hipertensão,maior triglicerídeos. $O$ estado civil, dieta e frequência de exercício eram diferenciais para estado de saúde.

\section{Palavras-chave:}

Diabetes tipo II, síndrome metabolica, fatores socio-demográficos.

\section{Introdução}

Muitos estudos sobre obesidade focam a população feminina, na qual a multimorbidade é mais frequentemente encontrada, visto que tende a ser a população que procura mais precoce e frequentemente serviços de saúde. Outros estudos mostram prevalência da obesidade e pior quadro de saúde na população masculina, com inicio mais precoce de obesidade, dislipidemia e hipertensão. Tem-se observado melhora geral da combinação dieta-exercício em termos de resultados, do que apenas dieta ou exercício, inclusive quanto à sensibilidade a insulina. A conservação dos ganhos para a saúde, após alguns anos, de acordo com a literatura, não se mantém, em função de atitudes inadequadas que tendem a ser retomadas.

O objetivo do presente estudo foi verificar a relação entre dieta, exercício, saúde, renda, escolaridade e estado civil, com obesidade, síndrome metabólica, diabetes do tipo dois, dislipidemia, hipertensão, glicemia e hemoglobina glicada (hbgli) em população masculina.

Amostra composta por 48 sujeitos selecionados em ambulatório de síndrome metabólica, sendo parte dos dados fornecidos pelos participantes e outra, coletada em prontuários.

\section{Resultados e Discussão}

A idade não se mostrou relevante nesta amostra, embora se saiba, via literatura, que tal influencia existe. Ser casado associava a maior hbgli, provavelmente por maior exposição à estimulação para alimentar-se, a qual os indivíduos não casados pareceram estar menos expostos. Apesar da média de idade ser semelhante, quando analisadas a escolaridade e a renda entre os que apresentavam hbgli $<6.5$ observou-se que a renda era menor, mas a escolaridade maior, bem como a frequência de exercícios, o que, contudo, não sugere controle da obesidade (II; sem dieta), hipertensão e dislipidemia. Os indivíduos com hbgli > 6,5 tinham maior renda e o mesmo padrão de escolaridade do grupo analisado anteriormente, mas alegavam fazer mais dieta e menos exercício. Apresentavam média de idade um pouco maior e tinham maior prevalência de dislipidemia e hipertensão. No grupo com hbgli $\geq 6,5$, observou-se baixo hdl, alto Idl, e menor frequência de exercício físico.Tinham alta glicemia e alegavam fazer dieta.O Grupo com hbgli <
6,5 apresentou mais triglicerídeos e baixo hdl, porém em uma zona mais intermediária (HDL: 30-44) e menor prevalência de hipertensão. Pacientes com síndrome metabólica apenas, apresentaram prevalência de zona intermediária de baixo HDL, LDL muito alto e maior prevalência de hipertensão que os demais grupos.

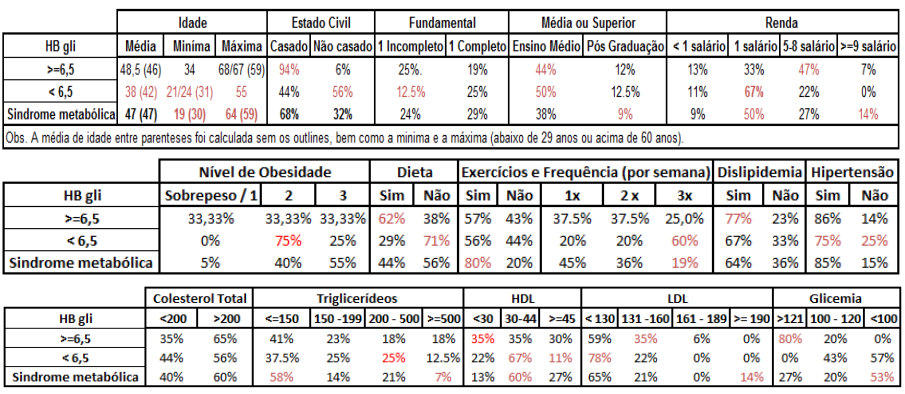

\section{Conclusões}

Havia associação de estado civil, renda e escolaridade com pior estado geral de saúde, no grupo com hbgli $\geq 6,5$; independe da média de idade do grupo: semelhante, entre tais pacientes e aqueles com hbgli $<6,5$ ou síndrome metabólica apenas. Apenas dieta, e baixa frequência de exercícios, aparentemente explicava tal situação. Com hbgli $<6,5$ havia prevalência de não casados, maior frequência de exercícios, mas sem dieta; havia associação com maior controle glicemia e hbgli, mas piores níveis de triglicerídeos. Assim, dieta e os exercícios físicos tinham diferentes repercussões na saúde dos pacientes. No grupo com síndrome metabólica não haviam associações do estado de saúde com idade, renda, dieta ou exercícios físicos. O diferencial era estado civil.

\section{Agradecimentos}

Serviço de Apoio ao Estudante (SAE); Prof. Dr. Marcos Antonio Tambascia (Chefe da disciplina de endocrinologia da FCM/Unicamp)

\footnotetext{
Goodpaster BH, Katsiaras A, Kelley DE Enhanced fat oxidation through physical activity is associated with improvements in insulin sensitivity in obesity.2003, Diabetes. ; 52(9):2191-7. Pedersen, J. M.:Lund, R.; Andersen, I.; Clark, A. J.; Prescott, E.; Rod, N. H.; Psychosocial risk factors for the metabolic syndrome: A prospective cohort study. International Journal of Cardiology. 2016, 215,41 46 Violán C, Foguet-Boreu Q, Roso-Llorach A, Rodriguez-Blanco T, Pons-Vigués M, Pujol-Ribera E Muñoz-Pérez MÁ, Valderas JM. Burden of multimorbidity, socioeconomic status and use of health services across stages of life in urban areas: a cross-sectional study.2014, BMC Public Health.14(530)1 13.

Villareal, D. T. , Chode, S, Parimi,N, Sinacore, D.R., Hilton,T., Armamento-Villareal, R.,Napoli,N., Qualls,C., Shah,K. Weight Loss, Exercise, or Both and Physical Function in Obese Older Adults, 2011, The New England Journal of Medicine, 364:1218-1229.
} 